\title{
TITLE:
}

\section{Noise reduction by dynamic signal preemphasis.}

\author{
$\operatorname{AUTHOR}(S)$ :
}

Takeda, Kazuyuki; Takegoshi, K

\section{CITATION:}

Takeda, Kazuyuki ...[et al]. Noise reduction by dynamic signal

preemphasis.. Journal of magnetic resonance 2011, 208(2): 305-308

\section{ISSUE DATE:}

2011-02

URL:

http://hdl.handle.net/2433/137555

\section{RIGHT:}

C 2010 Elsevier Inc.; この論文は出版社版でありません。引用の際には 出版社版をご確認ご利用ください。; This is not the published version. Please cite only the published version. 


\title{
Noise reduction by dynamic signal
}

\section{preemphasis}

\author{
Kazuyuki Takeda ${ }^{\mathrm{a}, *}, \mathrm{~K}$. Takegoshi ${ }^{\mathrm{a}}$, \\ ${ }^{a}$ Division of Chemistry, Graduate School of Science, Kyoto University, 606-8502 \\ Kyoto, Japan
}

\begin{abstract}
In this work we propose an approach to reduce the digitization noise for a given dynamic range, i.e., the number of bits, of an analog to digital converter used in an NMR receiver. In this approach, the receiver gain is dynamically increased so that the free induction decay is recorded in such an emphasized way that the decaying signal is digitized using as many number of bits as possible, and at the stage of data processing, the original signal profile is restored by applying the apodization that compensates the effect of the preemphasis. This approach, which we call APodization after Receiver-gain InCrement during Ongoing sequence with Time (APRICOT), is performed in a solid-state system containing a pair of ${ }^{13} \mathrm{C}$ spins, one of which is fully isotopically labeled and the other is naturally abundant. It is demonstrated that the exceedingly smaller peak buried in the digitization noise in the conventional approach can be revealed by employing APRICOT.
\end{abstract}

Key words: noise reduction, dynamic range, digitization noise, preemphasis, apodization 


\section{Introduction}

An analog to digital converter (ADC) is a device used to sample signals in the form of binary numbers suitable for data storage and processing on a computer. The error arising in the process of converting continuous analog signals to discrete binary numbers is known as the digitization noise. In the spectrum form, it causes the digitization sidebands spreading over the spectrum[1]. When an NMR spectrum of interest contains a single peak or peaks of comparable intensities, the effect of the digitization sidebands can be made negligibly small by amplifying the signal before digitization with an appropriate gain, because the digitization noise decreases as increasing the dynamic range of the signal, i.e., the number of bits used to represent the signal. Thus, the receiver gain is desirable to be large enough, but not to exceed the level causing ADC input overflow.

The digitization noise becomes appreciable when the spectrum consists of a set of peaks, one of whose intensity is exceedingly smaller than the others. In such a case, the digitization sidebands can conceal the smaller peak, because the receiver gain has to be set suitable to the largest intensity. One straightforward solution to this problem is to introduce a state-of-the-art NMR spectrometer equipped with the ADC with larger number of bits. In this work, we present an alternative approach to reduce the digitization noise for a given dynamic range of the ADC. By dynamically incrementing the receiver gain during acquisition of a free induction decay (FID) followed by application of apodization to the digitized signal to compenstae the different receiver gain

\footnotetext{
* corresponding author

Email address: takezo@kuchem.kyoto-u.ac.jp (Kazuyuki Takeda).
} 
for each data point, we show that the smaller peak buried in the digitization noise can be revealed. This strategy, which we call APodization after Receivergain InCrement during Ongoing sequence with Time (APRICOT), is an NMR analogue to noise reduction systems used in music recording on cassette tapes, in which dynamic preemphasis and deemphasis are employed during recording and playback. The present work may also remind the readers of previous efforts regarding to data sampling for improving the quality of NMR spectra. They include nonuniform data sampling[2-5], triangular sampling[6], random sampling[7], matched accumulation, i.e., data sampling at a fixed rate but different amounts of signal averaging[8], and so on. The present work is distinct from these previous works, in the sense that the former improves the dynamic range of one-dimensional spectra, while the latter concern sensitivity and/or spectral resolution of multidimensional experiments.

\section{A simple example}

As an example describing the idea of APRICOT, let us consider a signal $S(t)$ given by

$$
S(t)=\exp \left[-\frac{\Delta^{2} t^{2}}{16 \ln 2}\right] .
$$

Fig. 1(a)-(c) show digital representations of $S(t)$ for $\omega=2 \pi \cdot 200 \mathrm{~Hz}$ with dynamic ranges of 4,8 , and 12 bit. Fourier transformation of $S(t)$ results in a spectrum with a Gaussian line shape with a width $\Delta$, accompanied with the digitization sidebands. The sideband intensities decrease with increasing the dynamic range, as depicted in Fig. 1(e)-(g). At a glance, the spectra with the dynamic ranges of 8 bit and 12 bit (Fig. 1(f),(g)) appear to be identical with each other; the digitization sidebands are hidden in the thickness of the 
line. However, the magnified view of the baseline reveals that the effect of the digitization sidebands is smaller for the higher dynamic range.

Let us now suppose that the signal $S(t)$ is preemphasized so that the recorded data is constant over acquisition, and the data is then apodized using single precision calculation to restore the original signal profile (Fig. 1(d)). The spectrum obtained in this way has much less digitization sidebands, as shown in Fig. 1(h). The idea of APRICOT takes advantage of the fact that the number of bits used to process a number in a computer, typically 32 for single precision or 64 for double precision, is much larger than that of the conventional ADC, which is no more than 22 even for the latest commercial NMR spectrometer. In practical APRICOT experiments, the profile of the FID of interest determines the way that the receiver gain is dynamically manipulated, and the apodization function is applied accordingly to compensate the effect of preemphasis.

\section{Experimental}

For demonstration of APRICOT, we used an open-resource, field-programmable gate-array (FPGA) based NMR spectrometer, also called an OPENCORE NMR spectrometer $[9,10]$. A schematic diagram of the modified part of the spectrometer for this purpose is depicted in Fig. 3. The NMR signal was firstly amplified by a low noise amplifier to an appropriate level, then fed to one of two input ports of an analog RF multiplier AD834 (Analog Devices). The other input port received the gain control signal generated by a high speed 10 bit digital to analog converter (DAC) AD9740 (Analog Devices). The receiver gain was programmed with a pulse programmer of the spectrometer. Both the 
DAC and the pulse programmer operated at a clock frequency of $100 \mathrm{MHz}$. In this assembly, the receiver gain can be set in $2^{10}=1024$ steps and the time resolution is enough for the present purpose. The gain-modulated signal was digitized with an ADC AD9245BCPZ80 (Analog Devices) with a dynamic range of 14 bit and a sampling rate of $80 \mathrm{MHz}$.

Some NMR receivers, including the one used in the present study, employ a single high-speed ADC to record the FID in the form of an intermediate radiofrequency signal, and then demodulate the signal into the rotating frame representation by means of digital signal processing. On the other hand, many other NMR receivers sample the FID as a pair of demodulated audio signal components, namely, in-phase and quadrature components or often called real and imaginary components, using two separate ADCs. For the latter case, a pair of gain control circuits would be necessary for the individual ADCs.

In this work, ${ }^{13} \mathrm{C}$ CPMAS experiments were performed in a polycrystalline sample of $2-{ }^{13} \mathrm{C}$-labeled glycine in a magnetic field of $9.4 \mathrm{~T}$ using a Chemagnetics $3.2 \mathrm{~mm}$ T3 MAS probe. In this sample, the ${ }^{13} \mathrm{C}$ peak intensity for the natural abundance carboxyl carbon is expected to be two orders of magnitude smaller than that for the ${ }^{13} \mathrm{C}$-labeled methylene carbon.

\section{Results and Discussions}

Fig. 3(a) shows the in-phase and quadrature components of a ${ }^{13} \mathrm{C}$ CPMAS FID obtained with a constant gain in polycrystalline $2-{ }^{13} \mathrm{C}$-labeled glycine $(15 \mathrm{mg})$. Here, the signal was digitized and then demodulated by a digital module built inside the FPGA according to a procedure described in ref. [10]. These signal 
components were sampled every 10 us for $20.48 \mathrm{~ms}$, and those for the first 10 ms are plotted in the figure. Neither digital filtration nor signal accumulation was performed. Thus, the amplitude of the displayed signal reflects the input level of the signal at the ADC. Even though the dynamic range of the ADC was 14 bit, the receiver gain was set so that the maximum ADC input level at the beginning of the FID is around 13 bit for caution's sake to make sure that ADC overflow did not occur.

In the present case, the single-scan FID was relatively strong compared to the thermal noise level enough to show its decaying profile, thus allowing us to increase the receiver gain during acquisition without causing ADC overflow. Using the hardware described above, we performed the experiment in the same way except for the receiver gain, which was set at the same level at the beginning of the FID, and dynamically increased every $10 \mu \mathrm{s}$. Here, the receiver gain for the $k$ th data point was set to an integer nearest to $100 \cdot \exp \left(2.2 \times 10^{-3} k\right)$ $(k=1,2,3 \cdots)$, and total 950 steps were used until it was set stationary at the acquisition time of $9.5 \mathrm{~ms}$. The dynamic receiver gain and the corresponding apodization function are plotted in Fig. 3(b), and the preemphasized signal obtained in this way is shown in Fig. 3(c). This signal was then processed with a digital low-pass filter, and accumulated over 40 times. The resultant signal, plotted in Fig. 3(d), was finally apodized to compensate the preemphasized profile to obtain the signal shown in Fig. 3(e). For comparison, we also accumulated the conventional constant receiver-gain signals over 40 times.

The ${ }^{13} \mathrm{C}$ spectra obtained in the conventional way and with APRICOT are shown in Fig. 4(a),(b). For the methylene ${ }^{13} \mathrm{C}$ peak at $\sim 40 \mathrm{ppm}$, the resonance lines appear to be identical for both cases. On the other hand, the magnified view shows that the baseline noise level was considerably reduced in the case 
of APRICOT, revealing the the minor, natural abundance carboxyl ${ }^{13} \mathrm{C}$ peak at $\sim 180 \mathrm{ppm}$. Also, the peak intensity of the carboxyl carbon was found to be larger in the spectrum with APRICOT. This indicates that the receiver gain used in the conventional acquisition was insufficient for this minor peak, and appreciable amount of its digitization sideband dissipated over the spectrum, whereas the APRICOT approach could record this relatively weak signal with less digitization error, gathering the digitization sidebands into the resonance line.

In summary, we demonstrated that the digitization error can be reduced by recording the signal in such a preemphasized way that the ADC uses as many available bits as possible throughout acquisition, and by applying apodization afterwards on a computer to reproduce the original signal profile. The effect of this operation is to realize more accurate digital signal representation than that in the conventional case with the constant receiver gain. Even though the thermal noise cannot be eliminated neither by this strategy nor by any other tricks performed on the receiver, the effect of the reduced digitization noise can be beneficial in the presence of the peaks with large difference in their intensities. Thus, the APRICOT approach presented here can find interests of not only NMR measurements using the ADC with a relatively low dynamic range, but also of demanding experiments, such as NMR metabolomics studies and magnetic resonance imaging. 


\section{Acknowledgment}

This work has been supported by the CREST and SENTAN programs of Japan Science and Technology Agency.

\section{References}

[1] J. Keeler, Understanding NMR spectroscopy, Second Edition, Wiley (2010).

[2] J.C.J. Barna, E.D. Laue, J. Magn. Reson. 75 (1987) 384.

[3] D. Rovnyak, C. Filip, B. Itin, A.S. Stern, G. Wagner, R.G. Griffin, J.C. Hoch, J. Magn. Reson. 161 (2003) 43.

[4] M.W. Maciejewski, H.Z. Qui, I. Rujan, M. Mobli, J.C. Hoch, J. Magn. Reson. $199(2009) 88$.

[5] M.R. Gryk, J. Vyas, M.W. Maciejewski, Progress in Nucl. Magn. Reson. Spectroscopy $\mathbf{5 6}$ (2010) 329.

[6] K. Aggarwal, M.A. Delsuc, Magn. Reson. Chem. 35 (1997) 593.

[7] J.C. Hoch, M.W. Maciejewski, B. Filipovic, J. Magn. Reson. 193 (2008) 317.

[8] A. Kumar, S.C. Brown, M.E. Donlan, B.U. Meier, P.W. Jeffs, J. Magn. Reson. 95 (1991) 1.

[9] K. Takeda, Rev. Sci. Instrum. 78 (2007) 033103.

[10] K. Takeda, J. Magn. Reson. 192 (2008) 218. 


\section{Figure Captions}

\section{Figure 1}

A Gaussian decay function given in Eq. (1) for $\Delta=2 \pi \cdot 200 \mathrm{~Hz}$ represented with dynamic ranges of (a) 4 bit, (b) 8 bit, and (c) 12 bit. The solid line in (d) was obtained by applying Gaussian apodization with a width of $2 \pi \cdot 200 \mathrm{~Hz}$ to a constant function shown in the broken line using single precision calculation. (e)-(h) are their respective Fourier-transformed spectra. The magnified views of the spectral baseline are shown in blue lines.

\section{Figure 2}

A block diagram for the hardware used to implement APRICOT. LNA denotes a low noise amplifier.

\section{Figure 3}

(a) In-phase (red line) and quadrature (green line) components of digitally demodulated ${ }^{13} \mathrm{C}$ FID obtained by CPMAS in polycrystalline $2-{ }^{13} \mathrm{C}$-labeled glycine. Data was recorded with a constant receiver gain. (b) Dynamically varying receiver gain used in this work (purple line) and the corresponding apodization function (blue line). (c) $\mathrm{A}^{13} \mathrm{C}$ FID obtained in the same way as in (a) except for the varying receiver gain according to the function plotted in (b). This preemphasized signal was then processed by a digital low-pass filter with a cutoff frequency of $50 \mathrm{kHz}$, and accumulated over 40 scans with a sampling interval of $10 \mu$ s and acquisition length of 2048 to obtain the data in (d). It was finally apodized to restore the original profile of the signal shown in $(\mathrm{e})$. 


\section{Figure 4}

${ }^{13} \mathrm{C}$ spectra in a polycrystalline sample of $2-{ }^{13} \mathrm{C}$-labeled glycine obtained (a) in the conventional approach, and (b) with APRICOT. (b) was obtained by Fourier-transforming the data in Fig. 3(e). The purple lines are the 100-fold magnified view of the spectra, 
(a)

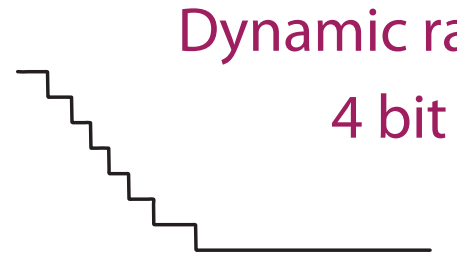

(b)

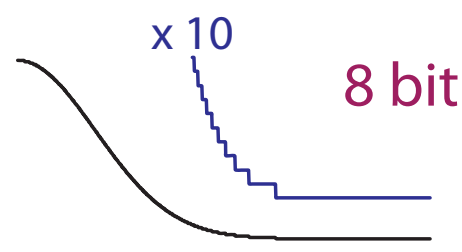

(c)

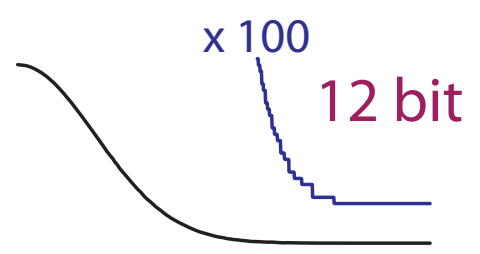

(d)

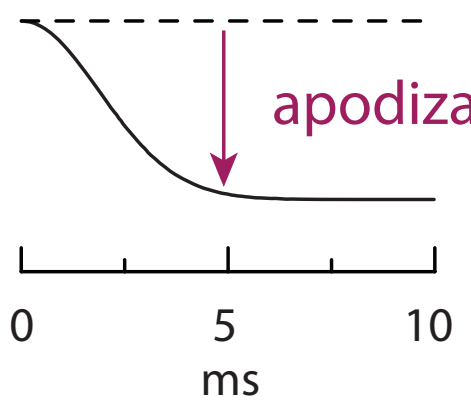

(e)

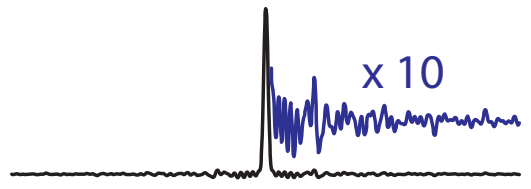

(f)

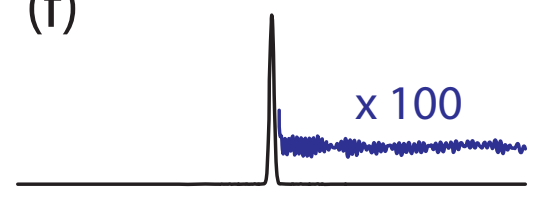

(g)

$\times 1000$

(h)

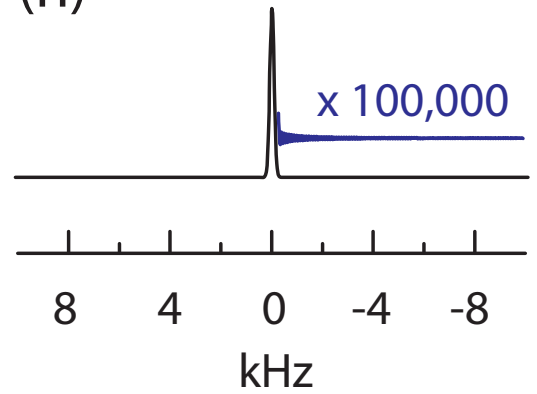

Fig. 1 


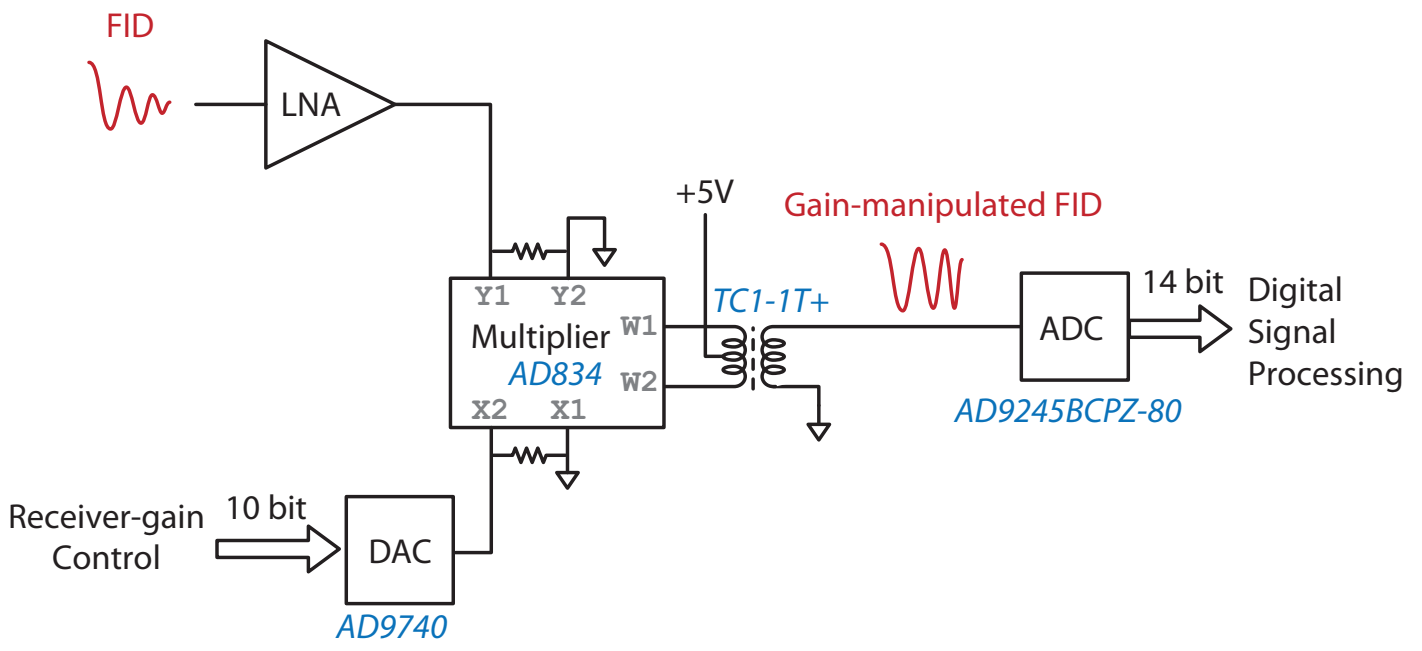

Fig. 2 
(a)

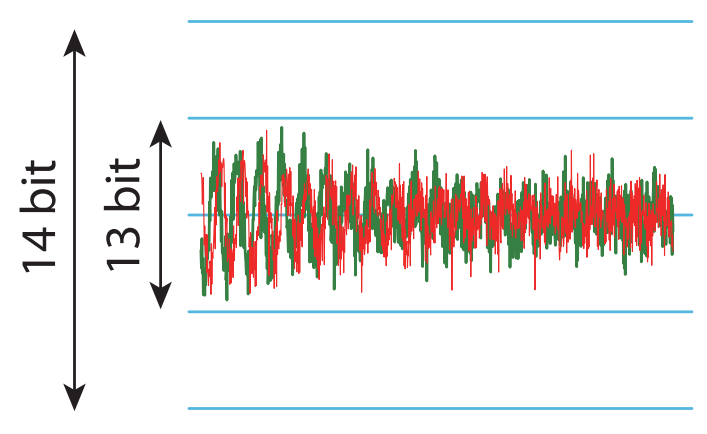

(c)
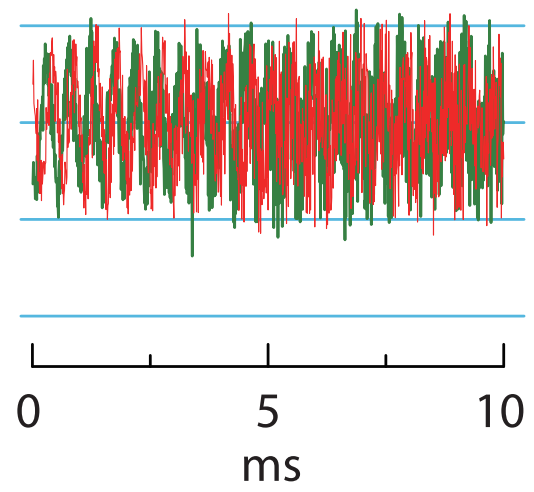

(b)

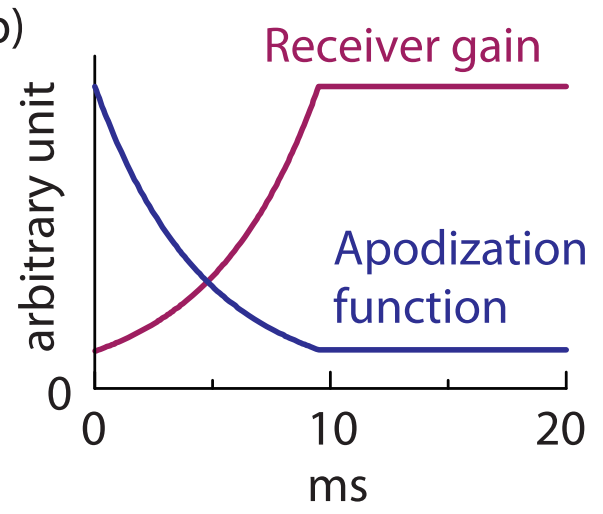

(d)

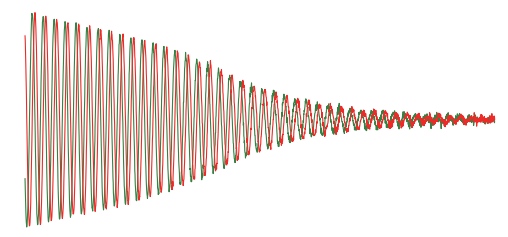

(e)
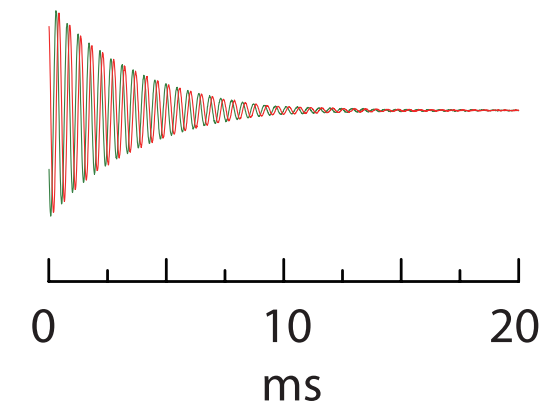

Fig. 3 
(a)

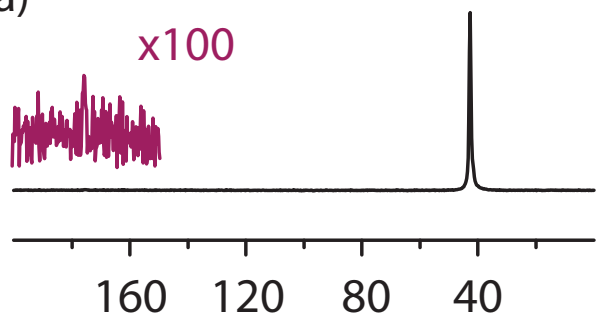

(b)

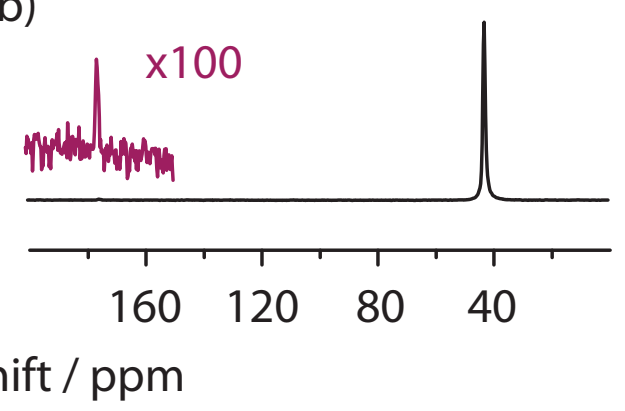

Fig. 4 